\title{
Metal hydride formation at pressures up to 1 Mbar
}

\author{
A Driessen $†$, P Sänger $\ddagger$, H Hemmes $†$ and R Griessen $\ddagger$ \\ $\uparrow$ University of Twente, Department of Applied Physics, Enschede, The Netherlands \\ $\ddagger$ Natuurkundig Laboratorium, Vrije Universiteit, Amsterdam, The Netherlands
}

Received 20 June 1990

\begin{abstract}
A simple mean-field lattice gas model is used to predict the hydride formation properties of $\mathrm{Cr}, \mathrm{Mn}, \mathrm{Fe}, \mathrm{Co}, \mathrm{Ni}, \mathrm{Mo}, \mathrm{Ru}, \mathrm{Rh}, \mathrm{Ir}, \mathrm{W}, \mathrm{Pt}, \mathrm{Cu}, \mathrm{Ag}$ and $\mathrm{Au}$, which at low hydrogen pressures absorb only small amounts of hydrogen. It is shown that with thermodynamic parameters determined from low-pressure data only (the molar standard heat of formation $\Delta \vec{H}_{x}^{0}$ and the Einstein temperature $\Theta_{\mathrm{E}}$ of the interstitial hydrogen vibration) it is also possible to describe their high-pressure behaviour. As a result, the pressure-composition isotherms of these 14 metals are given for several temperatures and for pressures up to $1 \mathrm{Mbar}$. Where possible, comparison is made with experimental data.
\end{abstract}

\section{Introduction}

There is a continuous progress in high-pressure technology for the formation of metal hydrides, especially with the increasing use of the diamond anvil cell [1-3]. For example, recently Hemmes and co-workers $[4,5]$ were able to load a Pd-Ag thin film at a hydrogen pressure of $80 \mathrm{kbar}$ and to monitor simultaneously the electrical resistance of the sample. For the correct interpretation of this kind of experiment one requires an adequate description of the thermodynamics of hydride formation at very high hydrogen pressure.

Metals which form hydrides at high hydrogen pressure, absorb hydrogen only poorly at low pressure. Because of the endothermic character of this reaction, hydrogen absorption measurements are usually carried out mainly at high temperatures [6-8]. Highpressure experiments are mostly done around room temperature [9-11]. For an adequate description of the hydride formation in both pressure and temperature regions, it would be very useful to have temperature- and pressure-independent parameters (e.g. the standard heat of formation) available. These parameters, in conjunction with the thermodynamic properties of hydrogen, allow us to describe completely the thermodynamics of hydride formation in the temperature and pressure range of interest.

We therefore decided to reanalyse existing experimental low-pressure data within a simple mean-field lattice gas model $[12,13]$ in order to obtain analytical expressions for the thermodynamic functions of hydrogen in a metal, such as the chemical potential $\mu_{\mathrm{H}}(P, T, x)$, the molar entropy $\bar{S}_{\mathrm{H}}(P, T, x)$ and the molar enthalpy $\bar{H}_{\mathrm{H}}(P, T, x)$. From these expressions the standard heat of formation $\Delta \bar{H}_{\infty}^{0}$, which is important e.g. for model calculations [14-16], can easily be derived. Together with the known thermodynamic properties of molecular hydrogen for pressures up to one megabar and temperatures up to $1000 \mathrm{~K}[17,18]$ complete pressure composition isotherms can be 
calculated $[12,13,19]$. It will be shown that, despite the simplicity of the model used and the fact that only low-pressure parameters enter the calculations, the results are in agreement with available experimental data at high pressures.

This paper is organized as follows. The thermodynamics of hydride formation at low and high pressure are given within the framework of a simple mean-field lattice gas model in section 2. For a meaningful description at high pressures one has to include, apart from the two low-pressure parameters $\Delta \bar{H}_{\infty}^{0}$ and $\Theta_{\mathrm{E}}$, the effect of the hydrogen concentration on the enthalpy and the effect of the non-zero partial molar volume of hydrogen in the hydride $\bar{V}_{\mathrm{H}}$. The concentration dependence of the enthalpy can lead to plateaux in the absorption isotherms below a critical temperature. At very high hydrogen pressure $V_{\mathrm{H}}$ may equal the molar volume of molecular hydrogen, and consequently further increase of hydrogen pressure will reduce the hydrogen content of the hydride. In the third section we derive the standard heat of formation for those poor hydrogen absorbing metals, for which sufficient experimental data are available (mainly work of McLellan and co-workers [20]). In section 4 we calculate the high-pressure-composition isotherms of 14 metal-hydrogen systems making use of the parameters $\Delta \bar{H}_{\infty}^{0}$ and $\Theta_{\mathrm{E}}$ as derived from low-pressure data, together with parameters taken from experimental data at high pressure (if available). Where possible, comparison with experimental data is made. The final section is a discussion of the results.

For the comparison with experimental hydride formation data at high pressure we use mainly the results of the groups of Baranowski [9] and Ponyatovskii [10,11]. There has been previous work on the formation of metal hydrides at very high pressures, see e.g. Fukai [21], Driessen and co-workers [12, 19], Griessen [22] and Hemmes and coworkers [13]; but here, for the first time, the exact thermodynamic functions of molecular hydrogen up to $1 \mathrm{Mbar}$ and up to $1000 \mathrm{~K}$ [17] have been used for a systematic study of the hydride formation behaviour of a large group of metals.

\section{Thermodynamics of hydride formation at high pressures}

We consider the hydride formation reaction

$$
\mathrm{M}+\frac{1}{2} x \mathrm{H}_{2} \Leftrightarrow \mathrm{MH}_{x}
$$

where $\mathrm{M}$ stands for metal and $x=[\mathrm{H}] /[\mathrm{M}]$. At thermodynamic equilibrium one has

$$
\frac{1}{2} \mu_{\mathrm{H}_{2}}(P, T)=\mu_{\mathrm{H}}(P, T, x) .
$$

The chemical potential of molecular hydrogen, $\mu_{\mathrm{H}_{2}}(P, T)$, has been determined by Hemmes and co-workers [17] for pressures up to $1 \mathrm{Mbar}$ and temperatures between 100 and $1000 \mathrm{~K}$. The chemical potential of hydrogen in the hydride, $\mu_{\mathrm{H}}(P, T, x)$, at a certain pressure $P$ and temperature $T$ can be related to the chemical potential at a reference pressure $P_{0}\left(P_{0}=1 \mathrm{~atm}\right.$. for convenience $)$ by means of the following relation:

$$
\mu_{\mathrm{H}}(P, T, x)=\mu_{\mathrm{H}}\left(P_{0}, T, x\right)+\int_{P_{0}}^{P} \bar{V}_{\mathrm{H}} \mathrm{d} P .
$$

Using a simple mean-field lattice gas model, $\mu_{\mathrm{H}}\left(P_{0}, T, x\right)$ can be written as [12] $\mu_{\mathrm{H}}\left(P_{0}, T, x\right)=R T \ln \left(\frac{x}{\beta-x}\right)+h(x)+\frac{3}{2} R \Theta_{\mathrm{E}}+3 R T \ln \left[1-\exp \left(-\Theta_{\mathrm{E}} / T\right)\right]+P_{0} \vec{V}_{\mathrm{H}}$. 
Table 1. The number, $\beta$, of interstitial sites per metal lattice atom available for hydrogen.

\begin{tabular}{llll}
\hline & \multicolumn{3}{c}{ Crystal structure } \\
\cline { 2 - 4 } Type of site & BCC & FCC & HCP \\
\hline Octahedral & 3 & 1 & 1 \\
Tetrahedral & 6 & 2 & $5 / 3$ \\
\hline
\end{tabular}

The first term is the ideal configurational entropy, $R$ is the molar gas constant and $\beta$ the number of intersitial sites available for hydrogen per metal atom. Table 1 gives $\beta$ for the most common metal structures: BCC (body-centred cubic), FCC (face-centred cubic) and HCP (hexagonal close packed) for octahedral and tetrahedral sites [23]. The parameter $\beta$ can be derived from low-pressure measurements, i.e. at low concentration $x$, so that blocking of sites can be neglected. At high pressures and large concentrations, blocking effects should be taken into account. If one assumes that every hydrogen atom blocks exactly $\beta$ interstitial sites, then the configurational entropy becomes

$$
R T \ln \left(\frac{x}{\beta(1-x)}\right)
$$

and the maximum concentration will be $x=1$.

For the second term of equation (4) one has, at infinite dilution, $h(0)=\frac{1}{2} E_{\mathrm{B}}-E_{0}$, where $E_{\mathrm{B}}$, the binding energy of the hydrogen molecule, $=4.748 \mathrm{eV}$ [24], and $E_{0}$ is the energy gained by bringing one extra hydrogen atom into the metal. At finite concentration $h(x)=h(0)+f(x)$, where $f(x)$ describes the effective hydrogen-hydrogen interaction energy. In this energy the influence of elastic lattice deformation as well as the change in electronic properties due to hydrogen uptake are included. In the following we assume the simplest case of $f(x)=a x$.

In transition metals the parameter $a$ arises mainly from the attractive elastic interaction between the dissolved hydrogen atoms. This attractive interaction is responsible for phase separation and, consequently, for a plateau region in the pressure-composition isotherms below a certain critical temperature $T_{\mathrm{c}}$, which is given by [25]

$$
T_{\mathrm{c}}=-a / 4 R .
$$

This relation is used in section 4 to give an estimate for the parameter $a$ from experimental pressure-composition isotherms.

The third and fourth terms of equation (4) represent the contribution of optical phonons of the interstitial hydrogen to the chemical potential. $\Theta_{E}=\hbar \omega_{E} / k_{B}$ is the Einstein temperature corresponding to the vibrational frequency $\omega_{E}$ for a given site occupation. The last term gives the work done by the hydride on absorbing hydrogen. This term is negligible at the pressure $P_{0}$.

In writing equation (4) an oversimplification has been made for the temperature dependence of the chemical potential, which is related to the excess entropy. From palladium is it known that a significant contribution to the excess entropy can arise from the change in the electronic heat capacity of the metal upon the addition of hydrogen [26]. Besides this there is a change in the acoustical phonon spectrum in the host metal $[27,28]$. In this paper we consider it pointless to include further effects in the chemical 
potential: the amount of experimental data available for the metals of interest-highpressure hydrides-is too small to justify a model with more parameters. The lattice gas model described above is thus mainly a useful parametrization scheme.

The contribution of the integral $\int \bar{V}_{\mathrm{H}}(P) \mathrm{d} P$ to the chemical potential, equation (3), is small at low pressures, typically $0.17 \mathrm{~kJ} \mathrm{~mol}^{-1} \mathrm{H}$ at $1 \mathrm{kbar}$, but determines almost completely the behaviour at very high pressures (e.g. $P \simeq 1 \mathrm{Mbar}$ ). Experimental values for $V_{\mathrm{H}}(P)$ are only available for a few metals at relatively low pressures. An estimate for the high-pressure behaviour can be made on the basis of a semi-empirical model for the heat of formation of metal hydrides (Griessen [29]), which relates the heat of formation to the interatomic distances of the host lattice. Within this model, Griessen derived that

$$
\frac{\mathrm{d}(\ln B)}{\mathrm{d} P}+\frac{\mathrm{d}\left(\ln \bar{V}_{\mathrm{H}}\right)}{\mathrm{d} P}=\frac{3.83}{B}
$$

where $B$ is the bulk modulus at zero pressure of the host metal. It is common to use a linear approximation for the pressure dependence of the bulk modulus [30, 31]:

$$
B(P)=B_{0}+B^{\prime} P \text {. }
$$

From equations (6) and (7), after some calculation, one obtains for $\bar{V}_{\mathrm{H}}$ if $B^{\prime} \neq 0$ :

$$
\int_{P_{0}}^{P} \bar{V}_{\mathrm{H}}(P) \mathrm{d} P=\frac{B_{0}+B^{\prime} P_{0}}{3.83}\left[\left(\frac{B_{0}+B^{\prime} P}{B_{0}+B^{\prime} P_{0}}\right)^{3.83 / B^{\prime}}-1\right] \bar{V}_{\mathrm{H}}\left(P_{0}\right) .
$$

By means of standard thermodynamic relations one obtains from (3) and (4) for the enthalpy:

$$
\begin{aligned}
\bar{H}_{\mathrm{H}}(P, T, x)= & h(0)+a x+\frac{3}{2} R \Theta_{\mathrm{E}}+3 R \Theta_{\mathrm{E}} n_{\mathrm{BE}}(T) \\
& +\int_{P_{0}}^{P}\left(\bar{V}_{\mathrm{H}}(P)-\left.T \frac{\partial \bar{V}_{\mathrm{H}}}{\partial T}\right|_{P}\right) \mathrm{d} P
\end{aligned}
$$

where $n_{\mathrm{BE}}(T)=1 /\left[\exp \left(\Theta_{\mathrm{E}} / T\right)-1\right]$ is the Bose-Einstein distribution function. The second term under the integral is small and may be safely neglected. For the entropy one finds the following:

$$
\begin{aligned}
\bar{S}_{\mathrm{H}}(P, T, x)= & -R \ln [x /(\beta-\beta x)]-3 R \ln \left[1-\exp \left(-\Theta_{\mathrm{E}} / T\right)\right] \\
& +3 R\left(\Theta_{\mathrm{E}} / T\right) n_{\mathrm{BE}}(T) .
\end{aligned}
$$

The partial molar heat of solution and the molar excess entropy change corresponding to reaction (1) are given by

$$
\begin{aligned}
& \Delta \bar{H}(P, T, x) \equiv \bar{H}_{\mathrm{H}}(P, T, x)-\frac{1}{2} \bar{H}_{\mathrm{H}_{2}}(P, T) \\
& \Delta \bar{S}_{\mathrm{EX}}(P, T, x) \equiv \bar{S}_{\mathrm{H}}(P, T, x)+R \ln [x /(\beta-x)]-\frac{1}{2} \bar{S}_{\mathrm{H}_{2}}(P, T, x) .
\end{aligned}
$$

For the standard partial molar heat of solution at infinite dilution, i.e. $x=0, T_{0}=$ $298.15 \mathrm{~K}$ and $P_{0}=1 \mathrm{~atm}$, one obtains (neglecting the $P \bar{V}_{\mathrm{H}}$ term):

$$
\Delta \bar{H}_{\nsim}^{0}=h(0)+\frac{3}{2} R \Theta_{\mathrm{E}}+3 R \Theta_{\mathrm{E}} n_{\mathrm{BE}}\left(T_{0}\right)-\frac{1}{2} \bar{H}_{\mathrm{H}_{2}}^{0} .
$$

This quantity together with $\Theta_{E}$ will be derived from experimental low-pressure absorption data in section 3 . 


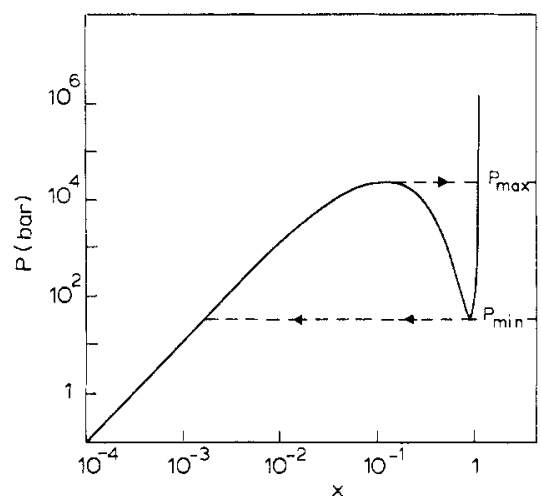

Figure 1. Schematic metal hydride formation isotherm. The broken lines give the highest absorption and lowest desorption isotherm in the plateau region.

Starting from the equilibrium condition (2) and assuming an ideal solution entropy, one can easily derive the following relation:

$$
\ln (x / \beta)=-\Delta \bar{H}(P, T) / R T+\Delta \bar{S}_{\mathrm{EX}}(P, T) / R
$$

for $x \ll 1$. Experimental results of solution at low concentration $x$ as a function of temperature at constant pressure are thus conveniently displayed as $\ln (x)$ versus $1 / T$. From the slope of such a plot, $\Delta \bar{H}(P, T)$ can be determined, and from an extrapolation of $1 / T \rightarrow 0, \Delta \bar{S}_{\mathrm{EX}}(P, T)$.

For the calculation of pressure-composition isotherms we proceed as follows. At thermodynamical equilibrium $\mu_{\mathrm{H}}(P, T, x)$ is completely determined through equation (2) by $\mu_{\mathrm{H}_{2}}(P, T)$. Using (3), (4) and (14), equation (2) can be cast in the following form:

$$
\frac{1}{2} \mu_{\mathrm{H}_{2}}(P, T)-\int_{P_{0}}^{P} \bar{V}_{\mathrm{H}}(P) \mathrm{d} P=g\left(\Delta \bar{H}_{x}^{0}, \Theta_{\mathrm{E}}, \beta, a, T, x\right)
$$

where all pressure-dependent terms appear in the left-hand side of the equation. At a given temperature $T$ and concentration $x$, with a suitable choice of $a$ and the parameters $\Delta \bar{H}_{x}^{0}, \Theta_{\mathrm{E}}$ and $\beta$ determined at low pressure, the right-hand side of $(16)$ can be calculated. Substituting equation (9) in (16) gives, for the left-hand side of this equation, a dependence on $T, P, B_{0}, B^{\prime}$ and $\bar{V}_{\mathrm{H}}\left(P_{0}\right)$. With the last three parameters taken from experiment (if possible) one is able by iteration to calculate the pressure $P$ at the given temperature $T$. There is an extra difficulty at very high pressures, when $\bar{V}_{\mathrm{H}}>\frac{1}{2} V_{\mathrm{H}_{2}}$. In this case a double solution is possible. The calculation can be repeated for a number of temperatures and concentrations. The results then are conveniently plotted in the form of absorption isotherms on a $\ln (p)$ versus $\ln (x)$ scale.

Figure 1 shows schematically a calculated pressure-composition isotherm. At low pressure, $P \leqslant 100$ bar, hydrogen exhibits essentially ideal gas behaviour. One can derive $[16]$ in that case from equations (3) and (4) for $x \ll 1$ :

$$
\frac{1}{2} \ln \left(P / P_{0}\right)=\ln x+\text { constant } \times T
$$

i.e. in a $\ln (P)$ versus $\ln (x)$ plot the isotherms can be represented by straight lines with slope 2 independent of the host metal and temperature.

At higher concentration and temperatures below $T_{c}$ there is a phase separation analogous to that occurring in a Van der Waals gas-liquid mixture. The calculated isotherms display unphysical behaviour in the two-phase region, where the hydride 
segregates into a hydrogen-poor and a hydrogen-rich phase. Because of metastability of the phases the absorption isotherm differs from the desorption isotherm. In the most extreme cases the isotherms follow the broken lines in figure 1.

\section{Determination of the standard molar heat of formation of some metal hydrides from experimental data}

In the previous section we presented a simple model for the thermodynamics of hydrogen absorption of metals. Within this model, all thermodynamic functions can be expressed in terms of the Einstein frequency $\Theta_{\mathrm{E}}$, the number of interstitial sites per metal atom $\beta$ and the function $h(x)$. By means of equations (10) and (14), $h(x)$ is related to the standard heat of formation $\Delta \bar{H}^{0}(x)$, which for infinite dilution, i.e. $x \ll 1$, reduces to $\Delta \bar{H}_{\infty}^{0}$. In the following we determine numerical values for the three parameters $\Delta \bar{H}_{\infty}^{0}, \Theta_{\mathrm{E}}$ and $\beta$ for a large group of poorly absorbing metals. This is done in three steps.

(i) Data input. From experimental data available in the literature we select a representative number of data points $x(P, T)$, within a temperature range as large as possible. There is a special difficulty involved with solubility data below $x=0.001$, where systematic errors can easily occur because of trapping at grain boundaries, voids or impurities (in particular oxygen), or errors due to adsorption at surfaces. Kiuchi and McLellan performed an extensive study of possible sources of discrepancy in the data of $\mathrm{Ni}, \mathrm{Co}, \mathrm{Pt}[8]$ and, in great detail, $\mathrm{Fe}$ [32]. They concluded that either only data above $600^{\circ} \mathrm{C}$ can be trusted, or, at lower temperatures, one should be sure to deal with very clean surfaces of the absorbing material. This means that data taken with material electrochemically polished or handled under UHV conditions should be preferred.

(ii) Data analysis. Starting with a data point $x(P, T)$, the chemical potential $\mu_{\mathrm{H}}(P, T, x)$ can be determined from (2). Then from (4), in which the number $\beta$ of interstitial sites is taken from experiment, if possible, or estimated from the metal lattice structure and table 1 , one obtains a relation between $\Theta_{\mathrm{E}}$ and $h(x)$. As the experimental data used are only at low concentration and at moderate pressure, we may simplify equation (4) by putting $h(x)=h(0)$ and $P \bar{V}_{\mathrm{H}}=0$, without introducing a significant error. For each choice of $\Theta_{E}$ we can then calculate a value for $\Delta \bar{H}_{\nsim}^{0}$ by means of (14). The final parameters $\Theta_{E}$ and $\Delta \bar{H}_{x}^{0}$ are determined from the complete set of data points using a variational method by minimizing the variance in $\Delta \bar{H}_{\nsim}^{0}$ with respect to a free varying $\Theta_{E}$. In table 2 the results for 14 metals are given.

(iii) Check of the analysis and comparison to experimental data. As most of the experimental data are given in the form of isobars, a check of the analysis can be made by calculating the 1 bar isobar on the basis of our set of thermodynamic parameters and comparing it with the experimental data (see figures 2 to 5 , later). If possible, in table 2 a direct comparison of our parameters is made with those derived in literature.

\subsection{Chromium, manganese and iron (figure 2)}

For chromium we use the data of Arnoult and McLellan [33] in the temperature range from 750 to $1350^{\circ} \mathrm{C}$. Chromium has a BCC structure; ion channelling measurements of Picraux and Vook [34] indicate that deuterium occupies octahedral positions in BCC $\mathrm{Cr}$. We therefore take $\beta=3$. 


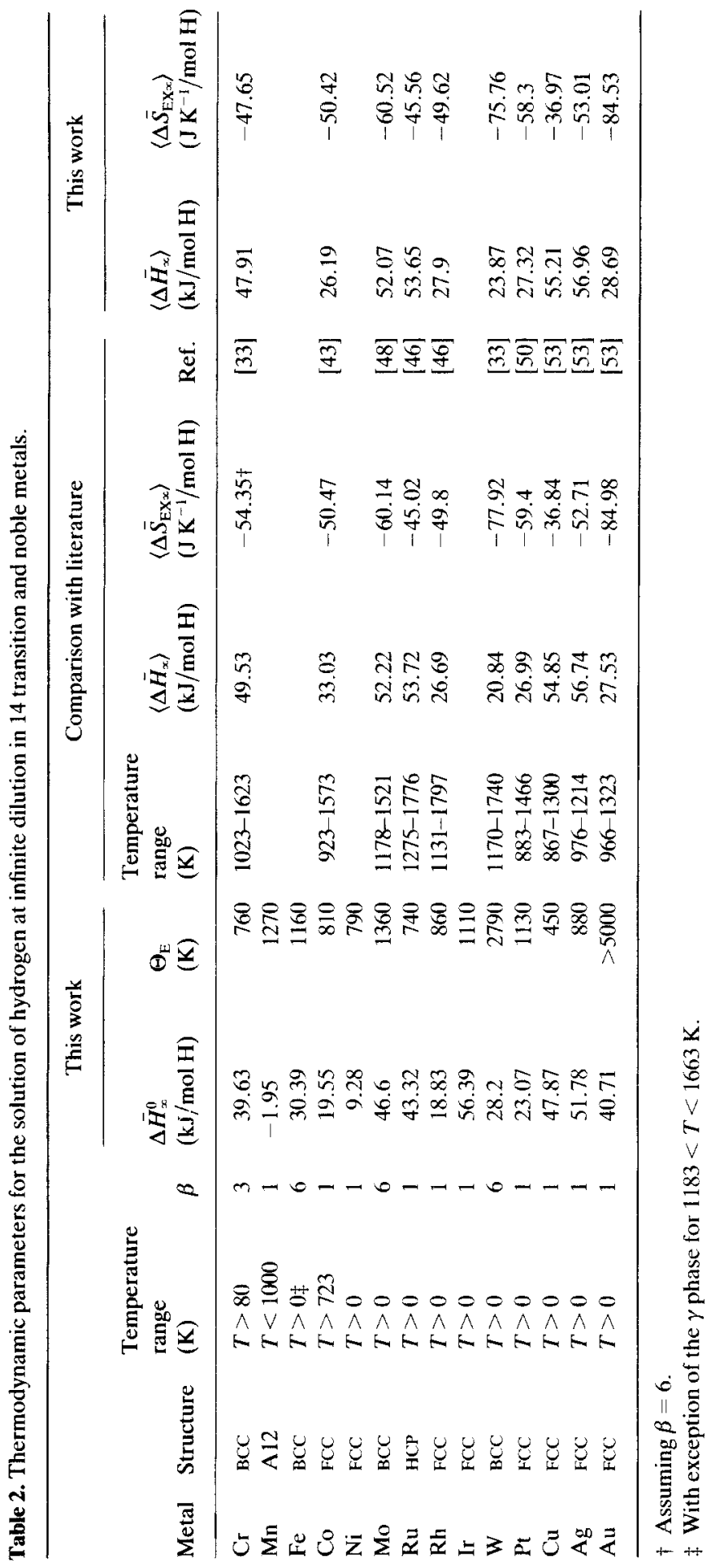




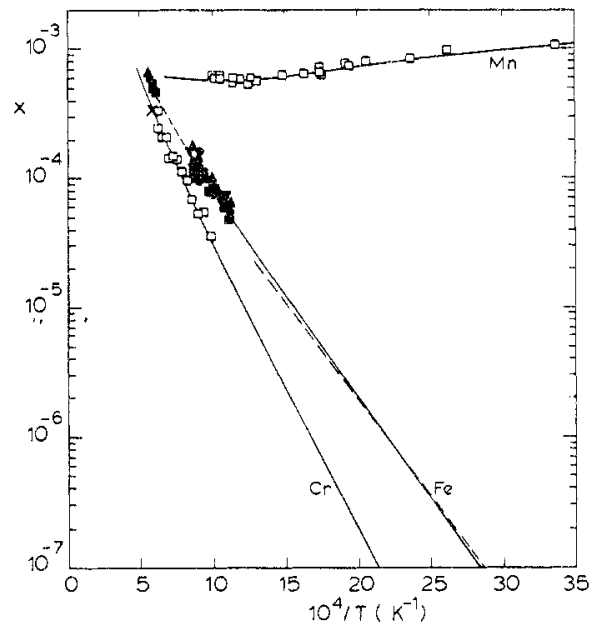

Figure 2. The 1 bar absorption isobars of $\mathrm{Cr}, \mathrm{Mn}$ and $\mathrm{Fe}$. Experimental points: $\mathrm{Cr}, \square[33]$; $\mathrm{Mn}, \square$ [35]; Fe, [37], [38]; $\mathbf{\nabla}[39], \mathbf{\Delta}[40], \times[41]$, - $[36]$. Full curves show our calculations with parameters from table 2 .

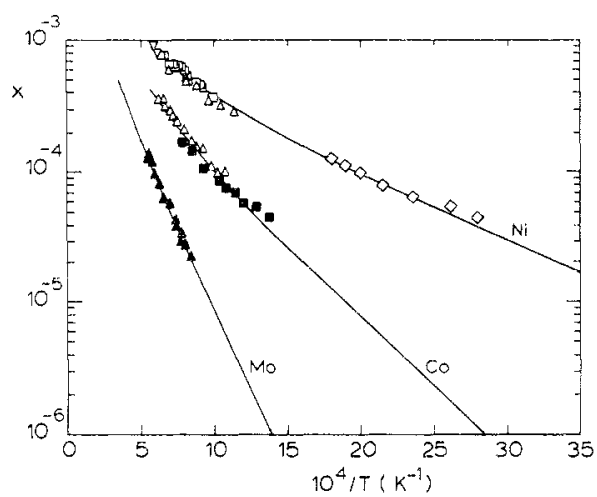

Figure 3. The 1 bar absorption isobars of $\mathrm{Co}, \mathrm{Ni}$ and Mo. Experimental points: Co, $\triangle[43], \mathbf{Q}[44]$; $\mathrm{Ni}, \triangle[43], \nabla[47], \square[46] ;$ Mo, $\Delta$ [48]. Full curves show our calculations with parameters from table 2.

In the case of manganese, with four solid phases, we restrict ourselves to the $\alpha$ phase, which is stable up to $727^{\circ} \mathrm{C}$ [35]. The experimental data of Potter and Lukens [35] show a weak minimum in the hydrogen absorption behaviour. It is difficult to assign a value to $\beta$, as $\alpha-\mathrm{Mn}$ has a complicated BCC A12 structure with 58 atoms per unit cell. For simplicity we assume $\beta=1$.

Because of its technical interest the hydrogen-iron system is very well studied in the low-concentration range (see [32] for a review). For the low-temperature region from 322 to $779 \mathrm{~K}$ we use the results of Quick and Johnson [36] obtained from permeability measurements in a UHV environment. Above $873 \mathrm{~K}$ up to the $\alpha \rightarrow \gamma$ phase transition at $1183 \mathrm{~K}$ the most recent data are those of Da Silva and co-workers [37] and Da Silva and McLellan [38]. We also include data by Martin [39] and Sieverts [40]. As the $\delta$ phase of $\mathrm{Fe}$ from $1663 \mathrm{~K}$ up to the melting line has the same $\mathrm{BCC}$ structure as the $\alpha$ phase, we assume a unique set of thermodynamical parameters for the absorption in both phases. The absorption data in the $\delta$ phase show fairly large disagreement. It is difficult to make a critical selection, and consequently we include all available data in our analysis, i.e. data by Da Silva and McLellan [38], Martin [39], Sieverts [40] and Luckemayer-Hasse and Schenk [41]. For the analysis we assume that hydrogen occupies the tetrahedral sites in the BCC $\alpha$ and $\delta$ phases as is suggested by calculations of Minot and Demangeat [42]. According to table 1 we therefore have $\beta=6$.

\subsection{Cobalt, nickel and molybdenum (figure 3)}

In the case of cobalt we restrict ourselves to the FCC high-temperature phase and use as input data above $600{ }^{\circ} \mathrm{C}$ the measurements of Stafford and McLellan [43] and Suzuki and McLellan [44]. For the FCC phase we expect hydrogen to occupy the octahedral sites, so that $\beta=1$. 


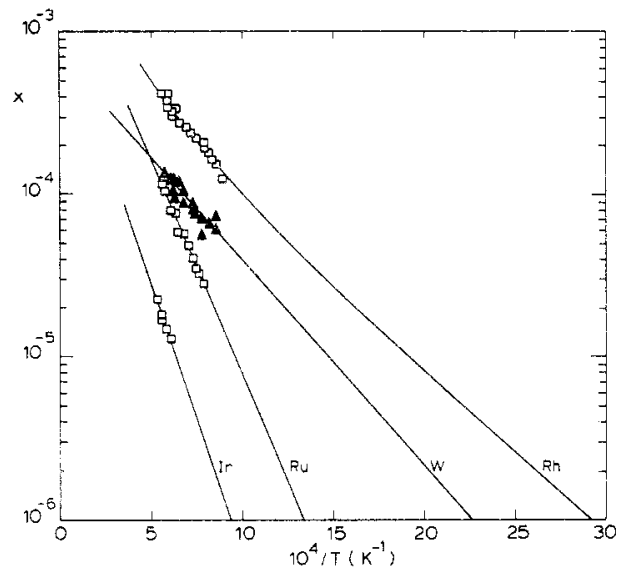

Figure 4. The 1 bar absorption isobars of $\mathrm{Ru}, \mathrm{Rh}$, Ir and W. Experimental points: Ru, Rh, Ir, $\square$ [46]; W, $\Delta$ [33]. Full curves show our calculations with parameters from Table 2 .

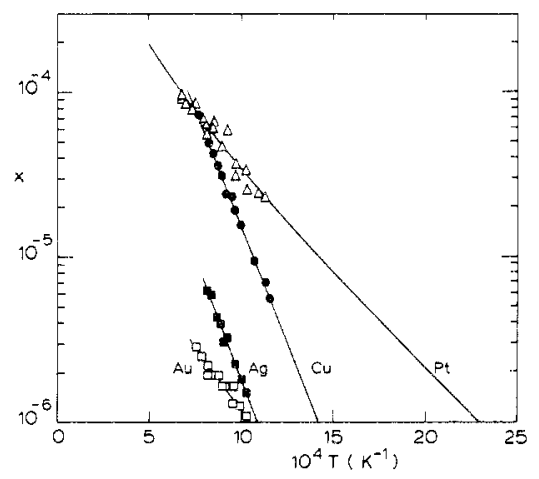

Figure 5. The 1 bar absorption isobars of $\mathrm{Pt}, \mathrm{Cu}$, $\mathrm{Ag}$ and $\mathrm{Au}$. Experimental points: $\mathrm{Pt}, \triangle[50] ; \mathrm{Cu}$, [53]; Ag, $\square$ [53]; Au, $\square$ [53]. Full curves show our calculations with parameters from table 2 .

There is much data on the hydrogen absorption of nickel (see [8] for a summary up to 1983), which especially at higher temperatures are consistent with each other, as can be seen in figure 3. For the low-temperature region (below $600^{\circ} \mathrm{C}$ ) the data of McLellan and Sutter [45] are probably more reliable as they pertain to electropolished samples. At temperatures higher than $600^{\circ} \mathrm{C}$ we use data points of Stafford and McLellan [43], McLellan and Oates [46] and Sieverts [47]. As Ni has the FCC structure, we assume that hydrogen occupies octahedral sites, i.e. $\beta=1$.

Molybdenum is a high-melting-point material with a $\mathrm{BCC}$ structure at all temperatures. The solubility of hydrogen in Mo is very low, and consequently the experimental data exhibit a large scatter. In our analysis we use the data of Oates and McLellan [48]. We assume hydrogen occupies the tetrahedral sites in Mo so that $\beta=6$.

\subsection{Ruthenium, rhodium, iridium and tungsten (figure 4)}

The absorption of hydrogen in $\mathrm{Ru}, \mathrm{Rh}$ and $\mathrm{Ir}$ is low. Only McLellan and Oates [46] present measurements, all of which were done at high temperature in order to have a measurable quantity of hydrogen absorbed in the highly endothermic reaction. We assume that for the FCC metals Rh and Ir, and for Ru with an HCP structure, hydrogen occupies octahedral sites $(\beta=1)$.

The absorption of hydrogen in $\mathrm{W}$, which is an especially poor hydrogen absorber, is measured by Arnoult and McLellan [33] in the temperature region from 897 to $1467^{\circ} \mathrm{C}$. $\mathrm{W}$ has a BCC structure at all temperatures. Ion channelling experiments on deuteriumimplanted W [49] reveal the expected tetrahedral interstitial position for the $\mathrm{D}$ atom, consequently $\beta=6$.

\subsection{Platinum, copper, silver and gold (figure 5)}

In the case of platinum, McLellan and co-workers have made the most recent determinations: a direct one, Yei and McLellan [50], and an indirect one by diffusion measurements (Katsuta and McLellan [51]). The diffusion method results in data typically 
one order of magnitude lower than the direct one. We will however use the direct determination, especially because of the agreement with Sieverts and Jurisch [52] at the highest temperature $(T \approx 1400 \mathrm{~K})$. As $\mathrm{Pt}$ has a FCC structure, we assume that hydrogen occupies the octahedral sites $(\beta=1)$.

The literature data on hydrogen absorption in $\mathrm{Cu}, \mathrm{Ag}$ and $\mathrm{Au}$ are controversial due to the very low solubility even at high temperatures. For our analysis we exclusively use the most recent data of McLellan [53], which is plotted in figure 5. All three metals have a FCC structure. Therefore hydrogen will probably occupy the octahedral sites $(\beta=1)$.

There is some difficulty with the results for gold and tungsten. Our analysis gives a very large ( $>5000 \mathrm{~K}$ and $2790 \mathrm{~K}$ respectively) Einstein temperature, which means that within the model used here, there is nearly no entropy involved. It could be that, with the very low amounts of hydrogen absorbed, one does not measure bulk effects of the pure material but rather adsorption on surfaces, trapping at impurities or effects due to grains, vacancies or voids. In this sense the $\Delta \bar{H}_{\varkappa x}^{0}$ values in table 2 for Au and $\mathrm{W}$ should be viewed as the lower bounds for the actually more endothermic heat of formation.

\section{Calculation of absorption isotherms}

Within the simple mean-field lattice gas model given above, the thermodynamical parameters of hydrogen absorption at low pressure and additional experimental data at high pressure (if available), one can calculate pressure-composition isotherms. The results are given graphically for a few temperatures in figures 6 to 19 , as we shall see. For the plateau region we apply Maxwell's construction.

The calculations are in principle only valid if the lattice structure of the host metal remains unchanged under hydride formation. With a change in structure one should work with different $\Delta \bar{H}_{\infty}^{0}$ and $\Theta_{\mathrm{E}}$ values for each structure. We have neglected this effect. In the experimental absorption isobars of $\mathrm{Fe}$ [37] or $\mathrm{Mn}$ [35], which have temperatureinduced phase transitions, one can see that these new phases introduce changes in $\Delta \bar{H}_{\nsim}^{0}$ of only a few kJ/mol $\mathrm{H}$. The estimate of $h(x)$ in equation (4), however, introduces possibly a larger error in the form of the isotherm at high concentrations.

In the following we present results of calculations of metal hydride formation isotherms for 14 metals. The parameters entering these calculations are given in table 3 .

\subsection{Chromium}

Chromium can electrolytically be loaded with hydrogen to form $\mathrm{HCP} \mathrm{CrH}$ or $\mathrm{FCC} \mathrm{CrH}_{2}$ [57]. From x-ray measurements the $\bar{V}_{\mathrm{H}}$ of $\mathrm{HCP} \mathrm{CrH}$ has been determined to be $1.29 \mathrm{~cm}^{3} \mathrm{~mol}^{-1} \mathrm{H}$. The phase diagram of $\mathrm{Cr}-\mathrm{H}$ has been measured by Ponyatovskii and co-workers [11]. Extrapolating their experimental data to higher temperatures, one expects a $T_{\mathrm{c}}$ of $800 \mathrm{~K}$ and $P_{\mathrm{c}} \simeq 27 \mathrm{kbar}$. Figure 6 shows our calculated isotherms, which are based on low-pressure thermodynamical data. As the $x \sim \vee V$ relation is fulfilled up to 100 bar [6], the calculations up to 100 bar and $x<0.01$ represents the exact isotherms within the accuracy of the experimental data at 1 bar. At higher concentration, probably due to the $\mathrm{BCC} \rightarrow \mathrm{HCP}$ phase transition, there is a rather poor agreement with experimental data [11]. Evidently the HCP phase has a less endothermic $\Delta \bar{H}_{\infty}^{0}$ value with consequently isotherms at a lower pressure. But as long as one does not enter the twophase region (i.e. $P<14$ kbar for absorption) the isotherms should be reasonably accurate. 
Table 3. Parameters used for our calculation of the pressure-composition isotherms. The bulk modulus is taken from reference [31].

\begin{tabular}{|c|c|c|c|c|c|c|c|c|c|c|c|}
\hline \multirow[b]{3}{*}{ Metal } & \multicolumn{5}{|c|}{ Properties for $x \ll 1$} & \multicolumn{6}{|c|}{ Properties for $x \simeq 1$} \\
\hline & \multirow[b]{2}{*}{ Structure } & \multicolumn{2}{|c|}{$\begin{array}{c}\text { Bulk } \\
\text { modulus }\end{array}$} & \multirow[b]{2}{*}{$\begin{array}{l}\bar{V}_{\mathrm{H}} \\
\left(\mathrm{cm}^{3} \mathrm{~mol}^{-1}\right)\end{array}$} & \multirow[b]{2}{*}{ Ref. } & \multirow[b]{2}{*}{ Structure } & \multirow[b]{2}{*}{$x \max$} & \multirow[b]{2}{*}{ Ref. } & \multirow[b]{2}{*}{$\begin{array}{l}T_{\mathrm{c}} \\
(\mathrm{K})\end{array}$} & \multirow[b]{2}{*}{$\begin{array}{l}P_{c} \\
\text { (kbar) }\end{array}$} & \multirow[b]{2}{*}{ Ref } \\
\hline & & $\begin{array}{l}B_{0} \\
\text { (Mbar) }\end{array}$ & $B^{\prime}$ & & & & & & & & \\
\hline $\mathrm{Cr}$ & $\mathrm{BCC}$ & 1.9 & 4.8 & 1.29 & {$[6]$} & $\mathrm{HCP}$ & 1 & 11 & 800 & 27 & [11] \\
\hline $\mathrm{Mn}$ & $\mathrm{A} 12$ & 0.61 & 5.0 & 2.7 & [54] & HCP & 0.96 & 11 & 730 & 9.4 & [11] \\
\hline $\mathrm{Fe}$ & $\mathrm{BCC}$ & 1.7 & 5.3 & 1.75 & {$[55]$} & $\mathrm{HCP}$ & 0.81 & 24 & 550 & 50 & [11] \\
\hline $\mathrm{Co}$ & FCC & 1.92 & 4.6 & 3 & {$[11]$} & FCC & 1 & 11 & 650 & $80 \dagger$ & \\
\hline $\mathrm{Ni}$ & FCC & 1.87 & 5.3 & 1.7 & {$[56]$} & FCC & 1.25 & 11 & 660 & 17.5 & [11] \\
\hline Mo & $\mathrm{BCC}$ & 2.7 & 4.7 & 1.3 & {$[54]$} & $\mathrm{HCP}$ & 1.3 & 11 & 700 & 43 & [11] \\
\hline $\mathrm{Ru}$ & $\mathrm{HCP}$ & 3.2 & 6.8 & $1.7 \ddagger$ & & & 0.03 & 11 & $600 \S$ & & \\
\hline Rh & $\mathrm{FCC}$ & 2.72 & 4.6 & 1.4 & {$[54]$} & FCC & 1 & 23 & 550 & 48 & [11] \\
\hline Ir & $\mathrm{FCC}$ & 3.54 & 4.9 & $1.7 \ddagger$ & & & \pm 0 & 11 & $600 \S$ & & \\
\hline W & $\mathrm{BCC}$ & 3.2 & 4.4 & $1.7 \ddagger$ & & & \pm 0 & 11 & $600 \$$ & & \\
\hline $\mathrm{Pt}$ & FCC & 2.79 & 5.5 & $1.7 \ddagger$ & & & \pm 0 & 11 & $600 \S$ & & \\
\hline $\mathrm{Cu}$ & FCC & 1.4 & 5.6 & $1.7 \ddagger$ & & & \pm 0 & 11 & $<0$ & & \\
\hline $\mathrm{Ag}$ & FCC & 1.02 & 6.2 & $1.7 \ddagger$ & & & \pm 0 & 11 & $<0$ & & \\
\hline $\mathrm{Au}$ & FCC & 1.73 & 6.2 & $1.7 \ddagger$ & & & \pm 0 & 11 & $<0$ & & \\
\hline
\end{tabular}

+ Estimated, based on figure 4.11 of reference [11].

$\ddagger$ Estimated 'standard' value $\left(\bar{V}_{\mathrm{H}}=1.7 \mathrm{~cm}^{3} \mathrm{~mol}^{-1}\right)$.

$\S$ Estimated 'standard' value $\left(T_{c}=600 \mathrm{~K}\right)$.

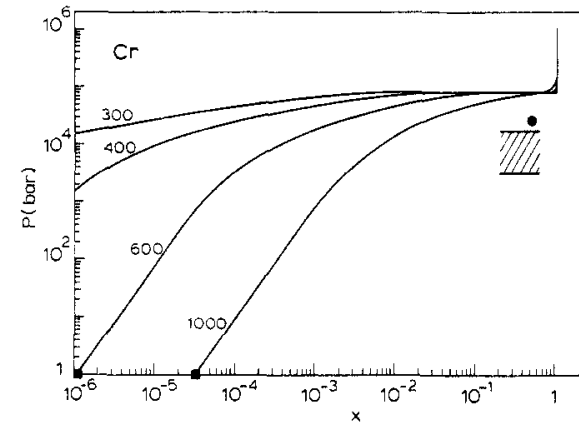

Figure 6. Metal hydride formation isotherms of $\mathrm{Cr}$. Full curves are our calculations with the temperature $(\mathrm{K})$ as a parameter; at $T=300 \mathrm{~K}, P_{\text {max }}=$ $100 \mathrm{kbar}$ and $P_{\min }=61 \mathrm{kbar}$. Experimental data: D, low-pressure data; $\bigcirc$, critical point $(800 \mathrm{~K}$, $27 \mathrm{kbar}$ [11]); the shading gives the plateau region at $423 \mathrm{~K}[9]$.

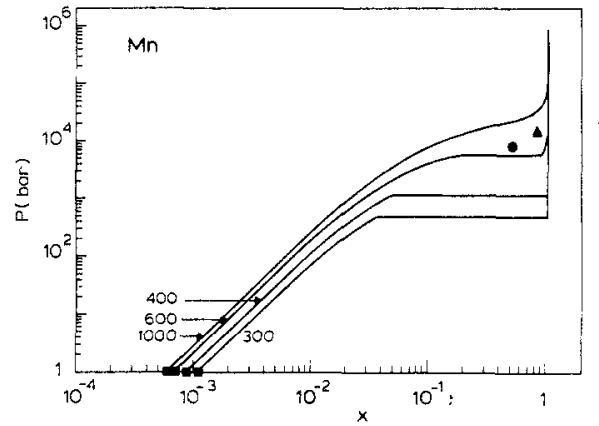

Figure 7. Metal hydride formation isotherms of $\mathrm{Mn}$. Full curves are our calculations with the temperature (K) as a parameter; at $T=300 \mathrm{~K}, P_{\max }=$ $1 \mathrm{kbar}$ and $P_{\min }=1.8$ bar. Experimental data: $\boldsymbol{\square}$, low-pressure data; , critical point $(730 \mathrm{~K}$, 9.4 kbar [11]); $\Delta$, from [9] $(573 \mathrm{~K}, 14 \mathrm{kbar}, x \simeq$ $0.8)$.

\subsection{Manganese}

Manganese can be loaded electrolytically with hydrogen [6]. With direct pressure loading, an HCP hydride can be formed, which is metastable at room temperature [11] 


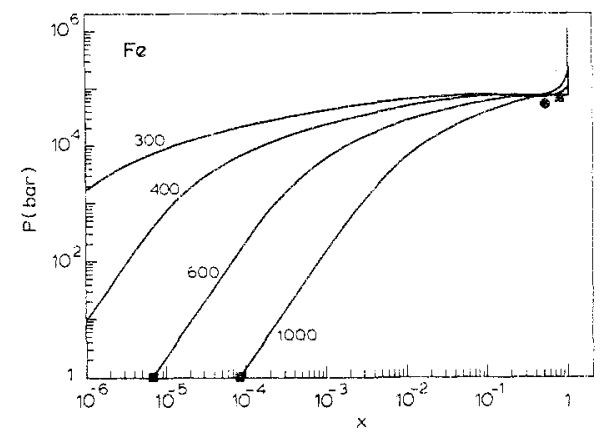

Figure 8. Metal hydride formation isotherms of Fe. Full curves are our calculation with the temperature $(\mathrm{K})$ as a parameter; at $T=300 \mathrm{~K}, P_{\text {nlax }}=$ $80 \mathrm{kbar}$ and $P_{\min }=64 \mathrm{kbar}$. Experimental data: $\mathbf{n}$, low-pressure data; $\boldsymbol{O}$, critical point $(550 \mathrm{~K}$, $50 \mathrm{kbar}[11]) ; \boldsymbol{\Delta}$, from [11] $(523 \mathrm{~K}, 67 \mathrm{kbar}$, $x=0.8$ ).

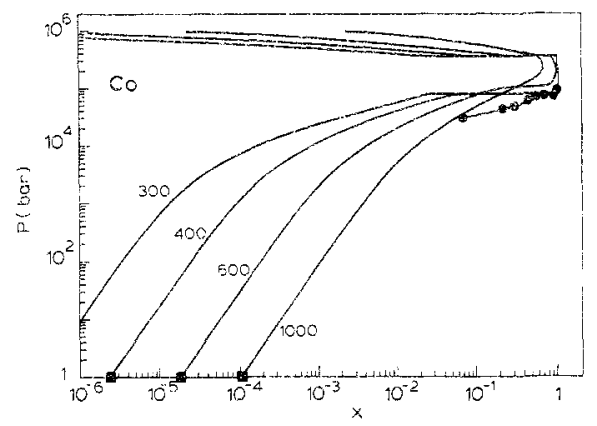

Figure 9. Metal hydride formation isotherms of Co. Full curves are our calculations with the temperature $(\mathrm{K})$ as a parameter; at $T=300 \mathrm{~K}, P_{\max }=$ $115 \mathrm{kbar}$ and $P_{\min }=40 \mathrm{kbar}$. Experimental data: , low-pressure data; $923 \mathrm{~K}$ absorption isotherm [11].

as is predicted by our calculation. The calculated isotherms, figure 7 , are in good agreement at low concentration, $x$, with somewhat less agreement at large $x$.

\subsection{Iron}

Iron with a phase transition from the BCC $\alpha$ phase to the HCP $\gamma$ phase at $1183 \mathrm{~K}$, forms, at pressures above $35 \mathrm{kbar}$, a hydride with an HCP lattice. At low pressures the relation $x \sim \vee P$ is fulfilled up to $140 \mathrm{~atm}$. [58]. Figure 8 displays our calculated isotherms, which at large concentrations show a slightly higher pressure in comparison with experimental data. Besides technical interest in the iron-hydrogen system, there are speculations that iron hydrides may have played an important role in the formation of the Earth's core [59].

\subsection{Cobalt}

Cobalt has an $\mathrm{HCP} \rightarrow$ FCC phase transition at approximately $450^{\circ} \mathrm{C}$ and ambient pressure. The thermodynamical parameters at infinite dilution and low pressure are only given for the high-temperature FCC phase. At high pressure, above $70 \mathrm{kbar}$, a FCC hydride is formed [11]. Our simple model cannot follow the peculiarities of the Co-H system in detail. From the experimental data [11] we cannot extract a critical temperature value, but as there is a broad plateau at $250{ }^{\circ} \mathrm{C}$, we estimate $T_{c} \simeq 650 \mathrm{~K}$. In figure 9 we give calculated isotherms which should be primarily valid for the FCC phase, but with less accuracy also for the HCP phase. There is reasonably good agreement with experiment.

Due to the large partial molar volume of hydrogen in $\mathrm{Co}\left(\bar{V}_{\mathrm{H}}=3 \mathrm{~cm}^{3} \mathrm{~mol}^{-1}\right)$, cobalt hydride becomes possibly unstable at very high pressures $(P>300 \mathrm{kbar})$, leading to a second two-phase region and plateau formation in the isotherms. This prediction has to be taken with great care, as at very high pressure the $P \bar{V}_{\mathrm{H}}$ term strongly determines the exact form of the isotherms, and $\bar{V}_{\mathrm{H}}$ has been extrapolated from low-pressure data. A 


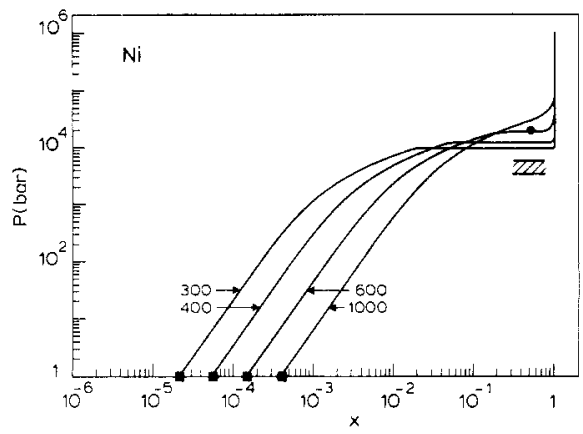

Figure 10. Metal hydride formation isotherms of Ni. Full curves are our calculations with the temperature $(\mathrm{K})$ as a parameter; at $T=300 \mathrm{~K}, P_{\max }=$ $15.5 \mathrm{kbar}$ and $P_{\min }=3.7 \mathrm{kbar}$. Experimental data: $\mathbf{n}$, low-pressure data; 0 , critical point $(660 \mathrm{~K}, 17.5 \mathrm{kbar}[11])$; shading gives the plateau region at $298 \mathrm{~K}$ [61].

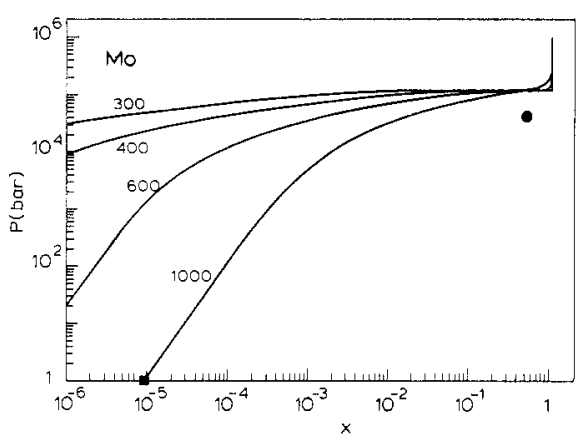

Figure 11. Metal hydride formation isotherms of Mo. Full curves are our calculations with the temperature (K) as a parameter; at $T=300 \mathrm{~K}, P_{\max }=$ $138 \mathrm{kbar}$ and $P_{\min }=108 \mathrm{kbar}$. Experimental data: $\square$, low-pressure data; 0 , critical point $(700 \mathrm{~K}$, 43 kbar) [11].

second plateau region, however, is thermodynamically allowed and can occur with a suitable set of parameters.

\subsection{Nickel}

Nickel is probably the best studied high-pressure hydride. Within the context of highpressure synthesis of metal hydrides it can be considered as a model system: easily accessible by high-pressure techniques $[11,56,60,61]$, no phase transition during hydride formation (both phases FCC), and reliable absorption data at low pressure [8]. Nickel hydride can be produced chemically or electrolytically, and is slow to decompose at room temperature. There is some experimental evidence that $\mathrm{Ni}$ forms hydrides with $x=1.25$ [11]. Probably hydrogen then occupies tetrahedral sites. Our calculation, however, only takes into account the octahedral sites. Our calculated isotherms, shown in figure 10 , are therefore restricted to $x \leqslant 1$. There is good agreement between the calculation and experiment at all pressures.

\subsection{Molybdenum}

Molybdenum undergoes a $\mathrm{BCC} \rightarrow \mathrm{HCP}$ phase transition during hydride formation. At high pressure $(P \simeq 70 \mathrm{kbar})$ it forms a stoichiometric hydride with $\bar{V}_{\mathrm{H}}=1.3 \mathrm{~cm}^{3} / \mathrm{mol} \mathrm{H}$ [54]. It cannot be produced electrolytically, and the hydride decomposes rapidly at room temperature. The $x \sim \vee P$ relation is fulfilled up to 100 bar [58]. Our calculated isotherms are given in figure 11 . They show systematically too high a pressure at high concentration.

\subsection{Ruthenium, iridium and platinum}

Ponyatovskii and co-workers $[11,62]$ measured the electrical resistance of these metals in hydrogen and in an inert pressure medium up to $90 \mathrm{kbar}$. With Pt and Ir there was no 


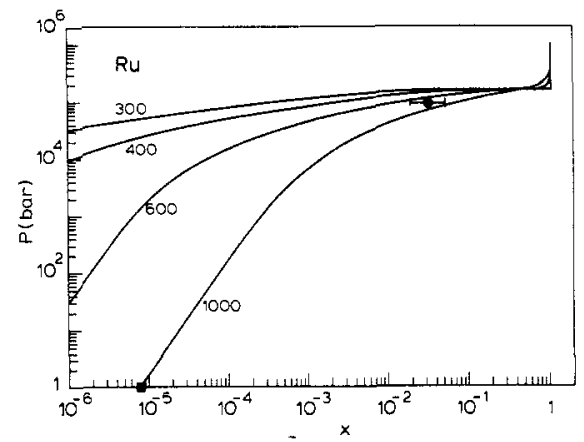

Figure 12. Metal hydride formation isotherms of Ru. Full curves are our calculations with the temperature $(\mathrm{K})$ as a parameter; at $T=300 \mathrm{~K}, P_{\max }=$ $173 \mathrm{kbar}$ and $P_{\min }=145 \mathrm{kbar}$. Experimental data: 口, low-pressure data; $\bigcirc$, from [11] (525 K, 90 kbar, $x \simeq 0.03$ ).

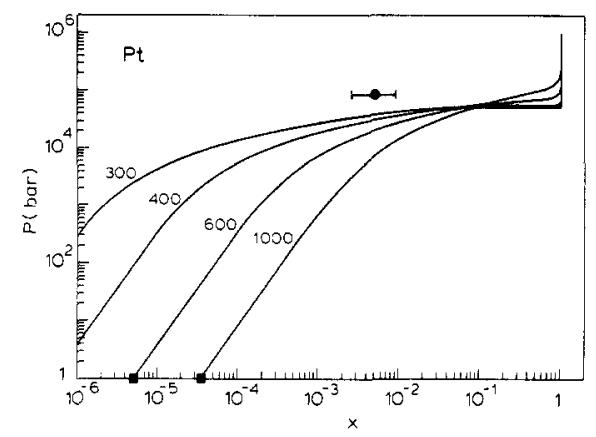

Figure 14. Metal hydride formation isotherms of Pt. Full curves are our calculations with the temperature (K) as parameter; at $T=300 \mathrm{~K}, P_{\text {max }}=$ $65 \mathrm{kbar}$ and $P_{\min }=45 \mathrm{kbar}$. Experimental data: $\mathbf{a}$, low-pressure data, $\bigcirc$, from [11] $(523 \mathrm{~K}$, 90 kbar, $x \simeq 0.005$ ).

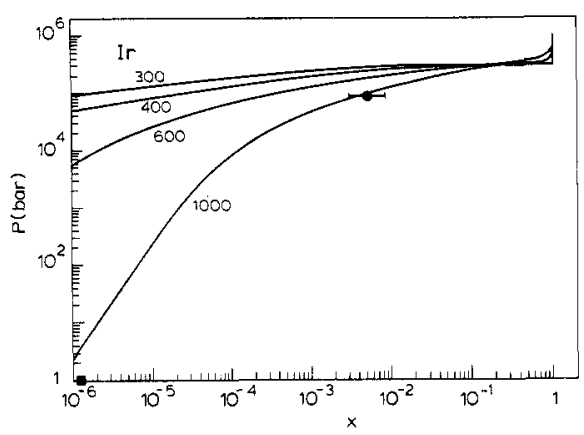

Figure 13. Metal hydride formation isotherms of Ir. Full curves are our calculations with the temperature $(\mathrm{K})$ as a parameter; at $T=300 \mathrm{~K}, P_{\max }=$ $325 \mathrm{kbar}$ and $P_{\min }=275 \mathrm{kbar}$. Experimental data: $\square$, low-pressure point $(1100 \mathrm{~K}, 1$ bar $)$; $\mathbf{O}$, from [11] $(523 \mathrm{~K}, 90 \mathrm{kbar}, x \simeq 0.005)$.

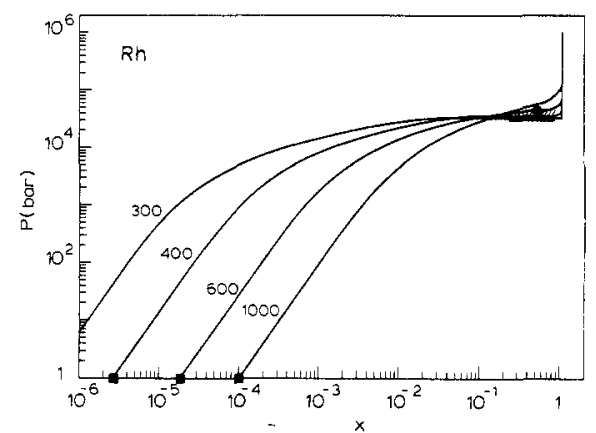

Figure 15. Metal hydride formation isotherms of $\mathrm{Rh}$. Full curves are our calculations with the temperature (K) as a parameter; at $T=300 \mathrm{~K}, P_{\max }=$ $40 \mathrm{kbar}$ and $P_{\min }=30 \mathrm{kbar}$. Experimental data: $\square$, low-pressure data; 0 , critical point $(550 \mathrm{~K}$, $48 \mathrm{kbar}[11]$ ); shading gives the desorption plateau at $300 \mathrm{~K}[11]$.

measurable difference, for $\mathrm{Ru}$ they measured a change in resistance of approximately $20 \%$. The determination of $x$ at $90 \mathrm{kbar}$ and $250{ }^{\circ} \mathrm{C}$ gave, for $\mathrm{Pt}$ and $\mathrm{Ir}, x \simeq 0.005$ and for Ru, $x=0.03$. For the values of $T_{\mathrm{c}}$ and $\bar{V}_{\mathrm{H}}$ we assume typical transition metal values: $T_{\mathrm{c}}=600 \mathrm{~K}$ and $\bar{V}_{\mathrm{H}}=1.7 \mathrm{~cm}^{3} \mathrm{~mol}^{-1} \mathrm{H}$. The calculated isotherms are given in figure 12 $(\mathrm{Ru})$, figure $13(\mathrm{Ir})$ and figure $14(\mathrm{Pt})$. The calculations for $\mathrm{Ru}$ and $\mathrm{Ir}$ are in reasonable agreement with the experimental points at $90 \mathrm{kbar}$. For Pt the calculations predict hydride formation below $90 \mathrm{kbar}$ in disagreement with experiment. This might be due to the lack of accurate experimental data at low pressure $[50,51]$.

\subsection{Rhodium}

Rhodium hydride, with a FCC structure, has been synthesized by high-pressure loading [11] above 45 kbar. Figure 15 shows the result of our calculation, which is in good agreement with experiment. 


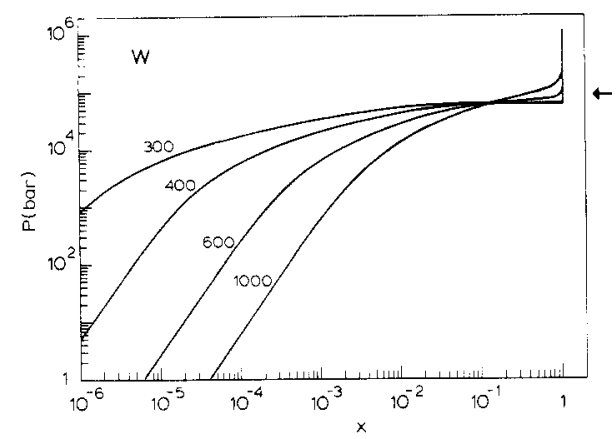

Figure 16. Metal hydride formation isotherms of W. Full curves are our calculations with the temperature $(\mathrm{K})$ as a parameter; at $T=300 \mathrm{~K}, P_{\max }=$ $72 \mathrm{kbar}$ and $P_{\min }=51 \mathrm{kbar}$. Experimental data: $\boldsymbol{\square}$, low-pressure data, arrow indicates the maximum experimental pressure with no measurable amount of hydrogen absorbed [11] for $T \leqslant 673 \mathrm{~K}$.

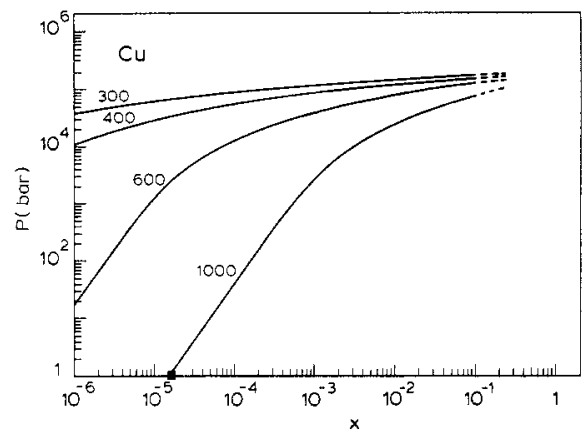

Figure 17. Metal hydride formation isotherms of $\mathrm{Cu}$. Full curves are our calculations with the temperature $(K)$ as a parameter. Experimental data: E, low-pressure data.

\subsection{Tungsten, copper, silver and gold}

Tungsten does not noticeably absorb hydrogen at pressures of $90 \mathrm{kbar}$ and $T \leqslant 400{ }^{\circ} \mathrm{C}$ [11]. The $x \sim \vee P$ relation is valid up to 100 bar [58].

Copper hydride can be synthesized chemically. It is fairly stable in water at low temperature. The formation of $\mathrm{AgH}$ and $\mathrm{AuH}$ by any means has not been reported yet. For $\mathrm{Cu}, \mathrm{Ag}$ and $\mathrm{Au}$ there are no data of successful hydrogen loading at high pressures. An estimate for the absorption behaviour at high pressures can indirectly be made from extrapolating the behaviour of $\mathrm{PdCu}, \mathrm{Pd}-\mathrm{Ag}$ or $\mathrm{Pd}-\mathrm{Au}$ alloys, which are studied extensively because of relatively high- $T_{\mathrm{c}}$ superconductivity $[4,11]$. In a local cluster approach for the local heat of formation of interstitial sites for hydrogen (see [13, 19, 22] for more details), one finds seven different surroundings $\mathrm{Pd}_{6-n} \mathrm{Ag}_{n}$ for an interstitial hydrogen atom in an octahedral site, depending on the nature of the six nearestneighbour metal atoms. One can show that the high pressure data currently available $(P \leqslant 90 \mathrm{kbar}[11])$ lead to a significant occupation with hydrogen only for clusters, which have at least two Pd atoms (i.e. $n \leqslant 4$ ). We therefore expect for hydride formation in $\mathrm{Cu}, \mathrm{Ag}$ or $\mathrm{Au}$ a hydrogen pressure substantially higher than $90 \mathrm{kbar}$.

As there are no experimental data of hydrides at high pressures for these four metals, we assume a standard value $\bar{V}_{\mathrm{H}}=1.7 \mathrm{~cm}^{3} / \mathrm{mol} \mathrm{H}$; for W we also assume $T_{\mathrm{c}}=600 \mathrm{~K}$. The resulting isotherms for $\mathrm{W}$ are given in figure 16 . In the case of the noble metals an estimate for the parameter $a$ (see equation (5)) can be made. In [13] Hemmes and coworkers give an expression for the $x$-dependence of the heat of formation; they find an elastic and an electronic contribution. The electronic contribution is related to $N\left(E_{\mathrm{F}}\right)$, the electronic density of states at the Fermi energy. Using this expression and values for $N\left(E_{\mathrm{F}}\right)$ from Moruzzi and co-workers [63], one finds for $a, 45,81$ and $50 \mathrm{~kJ} / \mathrm{mol} \mathrm{H}$ for $\mathrm{Cu}, \mathrm{Ag}$ and $\mathrm{Au}$ respectively. The calculated isotherms are given in figure $17(\mathrm{Cu})$, figure $18(\mathrm{Ag})$ and figure $19(\mathrm{Au})$. 


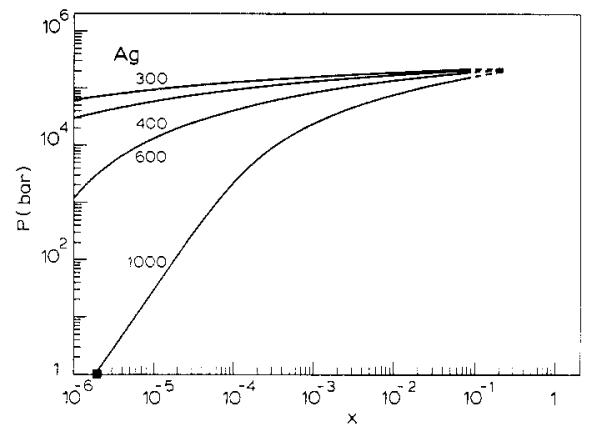

Figure 18. Metal hydride formation isotherms of Ag. Full curves are our calculations with the temperature (K) as a parameter. Experimental data: Q, low-pressure data.

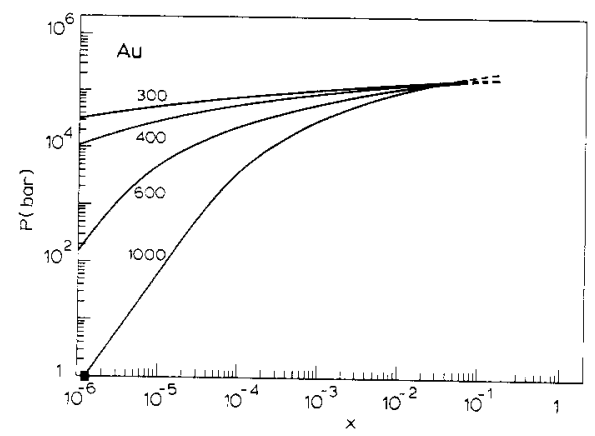

Figure 19. Metal hydride formation isotherms of Au. Full curves are our calculations with the temperature (K) as a parameter. Experimental data:

$\mathbf{\square}$, low-pressure data.

\section{Discussion}

In the foregoing we determined the thermodynamical parameters $\Delta \bar{H}_{\nsim}^{0}$ and $\Theta_{\mathrm{E}}$ for the hydrogen absorption of 14 late transition and noble metals from experimental data in the region of essentially infinite dilution. From a comparison of the experimental lowpressure data with calculated isobars in figures 2 to 5 it can be seen that the thermodynamical model described in section 2 is able to reproduce the experimental data within the experimental scatter. For some metals, McLellan and co-workers analysed the experimental data in terms of a mean $\Delta \bar{H}_{x}(T)$ and $\Delta \bar{S}_{\mathrm{EX} x}(T)$ for the temperature range of the experiments. Our analysis yields practically the same values - see table 2.

In section 4 we have presented pressure-composition isotherms for pressures up to $1 \mathrm{Mbar}$ for the same group of metals. It is interesting to note that nearly all metals show crossing of isotherms, i.e. the endothermic hydride formation at low pressures becomes exothermic at high pressures. The isotherms are most reliable at concentrations $x \leqslant 0.01$. In that case, we expect the accuracy to be as good as that of the experimental low-pressure absorption data. At larger concentrations the deviations can be more serious due to possible phase transitions and uncertainties in the hydride parameters $\bar{V}_{\mathrm{H}}$ and $h(x)$, which in our simple model is determined by $T_{\mathrm{c}}$. The uncertainty of the exact plateau pressure in hydrogen absorption and desorption experiments is inherent to the metastable character of hydride formation with large hysteresis in pressure. In our model the maximum hysteresis is determined by the interaction parameter $a$ or, with equation (5), by $T_{c}$. As the chemical potential of molecular hydrogen is growing less than linearly with pressure, the maximum hysteresis will grow with pressure, as can be seen in experiment and in our calculation. In the graphical representation of our calculation we emphasise the greater accuracy at low concentration and pressure by choosing a plot of $\log P$ versus $\log x$. The estimated error then is probably of constant magnitude over the entire logarithmic range of pressure and concentration.

Although the model used here is highly simplified and the parameters entering the model have only been determined from low-pressure data, the agreement between calculated and experimental data is relatively good, except with $\mathrm{CrH}$. The calculated isotherms may thus be used for estimates for hydride formation under extreme conditions. 
For some metals there is a need for experimental verification of hydride formation. Synthesis of most of the metal hydrides should be possible within the pressure range of the diamond anvil cell. Especially plateaux should easily be detected up to the Mbar range. The work presented here also needs an extension to the hydride formation of alloys. In the case of the Pd-noble metal alloys a cluster approach $[13,19,22]$ has been shown to give pressure-composition isotherms which are in good agreement with experiment.

\section{Acknowledgments}

This work was partially supported by the stichting voor Fundamenteel Onderzoek der Materie (FOM), which is financially supported by the Nederlandse organisatie voor Wetenschappelijk Onderzoek (NWO).

\section{References}

[1] Fukizawa A and Fukai Y 1983 J. Phys. Soc. Japan 522102

[2] Baranowski B, Hochheimer H D, Strössner K and Hönle W 1985 J. Less-Common Met. 113341

[3] Hemmes H, Driessen A, Kos J, Mul F A, Griessen R, Caro J and Radelaar S 1989 Rev. Sci. Instrum. 60 474

[4] Hemmes H, Driessen A, Rector J and Griessen R 1989 J. Phys.: Condens. Matter 18123

[5] Hemmes H 1989 PhD Thesis Vrije Universiteit, Amsterdam (copy available on request)

[6] Galaktionowa N A 1980 Hydrogen-Metal Systems Databook (Holon, Israel: Ordentlich)

[7] Mueller W A, Blackledge J P and Libowitz G G 1968 Metal Hydrides (New York: Academic)

[8] Kiuchi K and McLellan R B 1983 J. Less-Common Met. 95283

[9] Baranowski B 1978 Hydrogen in Metals II ed G Alefeld and J Völkl (Berlin: Springer) p 73

[10] Ponyatovskii E G, Antonov V E and Belash I T 1982 Sov. Phys. Usp. 25596

[11] Ponyatovskii E G, Antonov V E and Belash I T 1984 Problems in Solid State Physics ed A M Prokhorov (Moscow: Mir) p 109

[12] Driessen A, Hemmes H and Griessen R 1985 Z. Phys. Chem. N.F. 143145

[13] Hemmes H, Salomons E, Griessen R, Sänger P and Driessen A 1989 Phys. Rev. B 3910606

[14] Buschow K H J, Bouten P C P and Miedema A R 1982 Rep. Prog. Phys. 45927

[15] Griessen R and Driessen A 1984 Phys. Rev. B 304372

[16] Griessen R and Riesterer T 1988 Topics in Applied Physics 63: Hydrogen in Intermetallic Compounds I (Berlin: Springer) p 219

[17] Hemmes H, Driessen A and Griessen R 1986 J. Phys. C: Solid State Phys. 193571

[18] Hemmes H, Driessen A and Griessen R 1986 Physica 139 \& 140 B 116

[19] Driessen A, Hemmes H and Griessen R 1986 Physica 139 \& 140 B 694

[20] References are given in section 3

[21] Fukai Y and Sugimoto H 1983 Trans. Japan Inst. Met. 24733

[22] Griessen R 1986 Hydrogen in Amorphous and Disordered Solids ed G Bambakidis and R C Bowman (New York: Plenum) p 153

[23] King H W 1971 J. Mater. Sci. 61157

[24] Silvera I F 1980 Rev. Mod. Phys. 52393

[25] Lacher J R 1937 Proc. R. Soc. A 161525

[26] Oates W A and Flanagan T B 1971 Solid State Commun. 91841

[27] Magerl A, Stump N, Wipf H and Alefeld G 1977 J. Phys. Chem. Solids 38683

[28] Boureau G and Kleppa O J 1976 J. Chem. Phys. 653915

[29] Griessen R 1988 Phys. Rev. B 383690

[30] Walzer U 1987 Phys. Status Solidi b 140377

[31] Gerward L 1985 J. Phys. Chem. Solids 46925

[32] Kiuchi K and McLellan R B 1983 Acta Metall. 31961

[33] Arnoult W J and McLellan R B 1973 Acta Metall. 211397 
[34] Picraux S T and Vook F L 1974 Phys. Rev. Lett. 331216

[35] Potter E V and Lukens H C 1947 Trans. AIME 171401

[36] Quick N R and Johnson H H 1978 Acta Metall. 26903

[37] Da Silva J R G, Stafford S W and McLellan R B 1976 J. Less-Common Met. 49407

[38] Da Silva J R G and McLellan R B 1976 J. Less-Common Met. 501

[39] Martin E 1929 Arch. Eisenhüttenw. 3407

[40] Sieverts A 1911 Z. Phys. Chem. 77591

[41] Luckemayer-Hasse L and Schenk H 1932 Arch. Eisenhüttenw. 6209

[42] Minot Ch and Demangeat C 1987 J. Chem. Phys. 862161

[43] Stafford S W and McLellan R B 1974 Acta Metall. 221463

[44] Suzuki Y and McLellan R B 1986 Acta Metall. 34125

[45] McLellan R B and Sutter P L 1984 Acta Metall. 322233

[46] McLellan R B and Oates W A 1973 Acta Metall. 21181

[47] Sieverts A 1929 Z. Metall. 2137

[48] Oates W A and McLellan R B 1972 Scripta Metall. 6349

[49] Feldman L C, Mayer J W and Picraux S T 1982 Materials Analysis by Ion Channelling (New York: Academic)

[50] Yei W M and McLellan R B 1979 J. Less-Common Met. 6411

[51] Katsuta H and McLellan R B 1979 J. Phys. Chem. Solids 40697

[52] Sieverts A and Jurisch E 1912 Ber. Deutschen Chem. Gesell. 45221

[53] McLellan R B 1973 J. Phys. Chem. Solids 341137

[54] Somenkov V A, Glazkov V P, Irodova A V and Shilstein S Sh 1987 J. Less-Common Met. 129171

[55] Antonov V E, Belash I T, Degtyareva V F, Ponyatovskii E G and Shiryaev V I 1980 Sov. Phys.-Dokl. 25490

[56] Antonov V, Belash I T and Ponyatovskii E G 1977 Dokl. Akad. Nauk. 2331114

[57] Roy J and Gibb Th R P 1967 J. Inorg. Nucl. Chem. 29341

[58] Fromm E and Gebhardt E 1976 Gase und Kohlenstoff in Metallen (Berlin: Springer)

[59] Fukai Y 1984 Nature 308174

[60] Ponyatovskii E G, Antonov V E and Belash I T 1976 Dokl. Akad. Nauk. 229391

[61] Baranowski B 1972 Ber. Bunsenges. Phys. Chem. 76714

[62] Antonov V E, Belash I T, Malyshev V Yu and Ponyatovskii E G 1984 Platinum Met. Rev. 28158

[63] Moruzzi V L, Janak J F and Williams A R 1978 Calculated Electronic Properties of Metals (New York: Pergamon) 